\title{
Towards Participatory Design for Contextual Visualization in Education Using Augmented Reality X-ray
}

\author{
Marc Ericson C. Santos \\ Goshiro Yamamoto
}

Mitsuaki Terawaki

Jun Miyazaki

\author{
Graduate School of Information Science \\ Nara Institute of Science and Technology \\ 8916-5, Takayama, Ikoma, Nara, Japan, 630-0192 \\ \{chavez-s, mitsuaki-t, takafumi-t, goshiro, miyazaki, kato\}@is.naist.jp
}

\author{
Takafumi Taketomi \\ Hirokazu Kato
}

\begin{abstract}
We propose Augmented Reality (AR) $\mathrm{x}$-ray as an educational tool for contextual visualization-the presentation of virtual information in the rich context of a real environment. Teachers and students evaluated a state-of-the-art implementation of AR x-ray. Results show that realism, visibility, and perception of depth in AR x-ray are not significantly different from viewing $3 \mathrm{D}$ models with no occlusion cues. Moreover, teachers perceive AR x-ray useful.
\end{abstract}

\section{Categories and Subject Descriptors}

H.5.2 [Information Systems]: User Interfaces - interaction styles, prototyping, style guides, theory and methods, usercentered design.

\section{General Terms}

Design, Human Factors, Theory

\section{Keywords}

augmented reality, contextual learning, participatory design

\section{AR X-ray and Contextual Visualization}

Augmented Reality Learning Experiences (ARLEs) afford novel ways of interacting with information. AR x-ray is a specific kind of $\mathrm{AR}$ interaction wherein the user is given the illusion of looking through an occluded region. The challenge is to provide sufficient occlusion cues. Thus, techniques such as [1] are developed to decide which parts of the original scene should be kept, and which part should be replaced by virtual overlays.

The main application of AR x-ray is the visualization of the actual interior of a particular object. In designing ARLEs, we hypothesize that visualizing a conceptual abstraction of the interior of a target object is sufficient (i.e. we need not visualize the exact, actual interior of an object similar to the original concept of AR xray). AR x-ray uses contextual visualization wherein the real object is the context, and the interior of the object (virtual 3D models and/or virtually annotated symbols) is visualized.

\section{Augmented Reality X-ray Demonstration}

Using the method in [1], AR x-ray was implemented on iPad 2 tablets (dual core Apple A5, 512MB RAM, 32GB, 610 grams) using the back camera (480x640 pixel) and display (768x1024 pixel). ARToolkit was used to track the viewpoint and to interact with the virtual objects. We used a box ( object, and cultural artifacts as the virtual content to prevent suggesting educational applications to teachers.
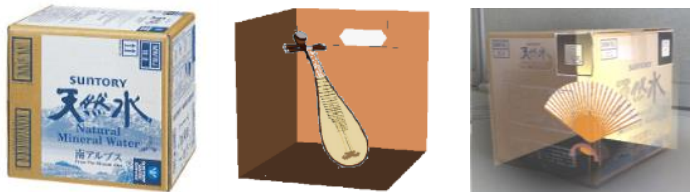

Figure 1: Object, 3D model and sample screenshot

We conducted a user study on 47 students (Filipinos, 21 male, 26 female, and aged 11-16). Participants were asked to rate the following statements on a five-point Likert scale: 1) The cultural artifact (3D model) seems real. 2) The cultural artifact is easy to see. 3) The cultural artifact is inside the box. The results show that realism, visibility and depth constructs in AR $\mathrm{x}$-ray are not different from viewing 3D models without occlusions.

Table 1. Summary of Realism, Visibility and Depth Scores

\begin{tabular}{|c|c|c|c|c|}
\hline \multirow{2}{*}{ Construct } & \multicolumn{2}{|c|}{$\begin{array}{c}\text { No Occlusion (3D model } \\
\text { is superimposed on box) }\end{array}$} & \multicolumn{2}{|c|}{$\begin{array}{c}\text { With Occlusion } \\
\text { (AR x-ray) }\end{array}$} \\
\cline { 2 - 5 } & $\begin{array}{c}\text { Mean } \\
\text { (95\% Conf.) }\end{array}$ & STDV & $\begin{array}{c}\text { Mean } \\
\text { (95\% Conf.) }\end{array}$ & STDV \\
\hline Realism & $3.1(2.6-3.7)$ & 1.2 & $3.0(2.5-3.5)$ & 1.3 \\
\hline Visibility & $3.8(3.3-4.3)$ & 1.1 & $4.0(3.5-4.4)$ & 1.1 \\
\hline Depth & $3.4(2.9-3.9)$ & 1.2 & $3.4(3.0-3.9)$ & 1.2 \\
\hline
\end{tabular}

Teachers expressed their interest in AR x-ray-based learning materials in a focus group discussion. They have identified two topics: plant processes (visualizing roots and seeds interior) and human internal anatomy. The teachers foresee that AR x-ray will have the advantage of allowing students to "learn by experience," which can improve their attention, and increase their motivation.

\section{REFERENCES}

[1] Sandor, C., Cunningham, A., Dey, A. and Mattila, V. An Augmented Reality X-Ray System Based on Visual Saliency, In IEEE ISMAR Proceedings, pp. 27-36, October 2010.

[2] Hull, D. M. Opening Minds, Opening Doors: The Rebirth of American Education, CORD, TE, USA, 1993. 